\title{
ParkCrowd: Reliable Crowdsensing for Aggregation and Dissemination of Parking Space Information
}

\author{
Fengrui Shi, Student Member, IEEE, Di Wu, Member, IEEE, Dmitri I. Arkhipov, Member, IEEE, \\ Qiang Liu, Member, IEEE, Amelia C. Regan, Member, IEEE, and Julie A. McCann
}

\begin{abstract}
The scarcity of parking spaces in cities leads to a high demand for timely information about their availability. In this paper, we propose a crowdsensed parking system, namely ParkCrowd, to aggregate on-street and roadside parking space information reliably, and to disseminate this information to drivers in a timely manner. Our system not only collects and disseminates basic information, such as parking hours and price, but also provides drivers insights of the real-time and future availability of parking spaces based on aggregated crowd knowledge. To improve the reliability of the information being disseminated, we dynamically evaluate the knowledge of crowd workers based on the veracity of their answers to a series of location-dependent point of interest (POI) control questions. We propose a logistic regression based method to evaluate the reliability of the crowd knowledge for real-time parking spaces information. Besides, a joint probabilistic estimator is employed to make inference of parking spaces' future availability based on crowdsensed knowledge. Moreover, to incentivise wider participation of crowd workers, a reliability based incentivisation method is proposed to reward workers according to their reliability and expertise levels. The efficacy of ParkCrowd for aggregation and dissemination of parking space information has been evaluated in both real-world tests and simulations. Our results show that the ParkCrowd system is able to accurately identify the reliability level of the crowdsensed information, estimate the potential availability of parking spaces with high accuracy, and be successful in encouraging participation of the more reliable crowd workers by offering them higher monetary rewards.
\end{abstract}

Index Terms-Smart parking, mobile crowdsensing, locationbased service, human reliability, incentivisation.

\section{INTRODUCTION}

W ITH the increasing numbers of automobiles in cities, finding a parking space which is close to one's driving destination is costly, time-consuming, and contributes to traffic congestion in urban areas [1]. These problems are especially extreme in the case of on-demand parking in metropolitan centres and densely populated areas around the world [2]. Therefore, there is a strong need to disseminate availability information of vacant parking spaces in real-time. Although

F. Shi is with the Department of Computing, Imperial College London, London SW7 2AZ, UK (e-mail: fengrui.shi14@imperial.ac.uk).

D. Wu is with the Department of Computer Science, University of California, Irvine, CA 92617, USA (e-mail: dwu3@ics.uci.edu).

D. I. Arkhipov is with the Department of Computer Science, University of California, Irvine, CA 92617, USA (e-mail: darkhipo@ics.uci.edu).

Q. Liu is with the Department of Computer Science, Dartmouth College, Hanover, NH 03755, USA (e-mail: qiang.liu@dartmouth.edu).

A. C. Regan is with the Department of Computer Science, University of California, Irvine, CA 92617, USA (e-mail: aregan@uci.edu).

J. A. McCann is with the Department of Computing, Imperial College London, London SW7 2AZ, UK (e-mail: j.mccann@imperial.ac.uk).

Manuscript received ...
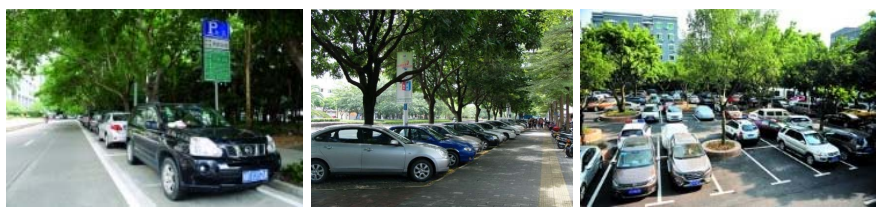

Fig. 1. Examples of on-street and roadside parking spaces.

drivers can view the locations of parking lots on maps offered by popular location based service (LBS) providers, e.g. Google or Baidu, some important information of nearby unoccupied on-street or off-street parking spaces such as their parking hours, prices as well as the potential availability level are missing from these providers due to the lack of an efficient approach for collecting this information in real-time. It is therefore necessary to design and implement a system for aggregating and disseminating the relevant information for these urban parking spaces.

\section{A. Motivation}

The scarcity of parking spaces in cities, especially metropolises such as London, New York and Beijing, leads to high demands for timely information revealing their availability during busy periods of the day. Although off-street parking garages are usually equipped with the necessary infrastructure or personnel to publish the number of unoccupied spaces [3], [4], [5], the lack of available spaces and high prices/access delays in these facilities force many drivers to alternative mechanisms such as using on-street and roadside parking spaces on demand (examples of such parking spaces are illustrated in Fig. 1). However, the lack of timely information about those kinds of parking spaces frustrates drivers, leading to an increase in congestion, and to unnecessary expenditures of time and money by the drivers looking for vacant spots.

The most straightforward way to provide such information is to publish it on the Internet. This can be done by the owner of the parking space [4]. However, this method doesn't suit scenarios such as free on-street parking and roadside parking lots as there is no incentive to publish this data. The data could be derived by constructing spatiotemporal models and applying them to historical data with geographic correlation [6], however the published data is usually outof-date and inaccurate for real-time demands. A number of urban parking systems rely on specialised infrastructure, either embedded in roads or in vehicles to capture real-time parking space occupancy [3], [5], [7], [8]. However, the cost to deploy these sensors or short-range communication technologies when scaling to large cities is significant. 


\section{B. Our Approach}

With the wide adoption of smartphones and other mobile devices, mobile crowdsensing (MCS) [9], [10], [11], an approach to outsource data collection tasks among crowd workers, could be a promising solution for the aggregation and dissemination of parking space information. However, recent research or commercial products mainly employ sensors based solutions [3], [12], [13], which are only able to detect the existence of parking spaces, but not other important information such as parking time, hourly price and whether it is privately-owned or public. Furthermore, these approaches are not able to estimate future availability information and thus hindering the drivers from planning their trip in advance based on future availability.

In this paper, we present ParkCrowd, which takes a "Human-in-the-loop" approach by utilising crowd workers to identify vacant parking spots and score future availability level based on their own predictions. Using the knowledge of crowd workers for parking spaces, ParkCrowd is able to not only provide the real-time availability information, but also offer insights on the future availability of parking spaces to help drivers plan their trips in advance. However, the reliability of the crowd workers' knowledge is highly uncertain and has big impact on the performance of ParkCrowd. In this paper, inspired by the location-dependent nature of parking information, we introduce point of interest (POI) related control questions into the crowdsensing process 1) to estimate the reliability of real-time availability information and 2) to improve the accuracy of estimated future availability. Moreover, to incentive crowd workers' participation as well as to encourage them to provide more reliable information, we build an incentivisation scheme into ParkCrowd to reward workers based on the reliability of contributed knowledge.

The major contributions of this paper are listed as follows:

- We design ParkCrowd, an MCS system for collecting and disseminating vacant parking space information based on crowd workers' knowledge.

- We build models to evaluate the reliability of the crowd workers' knowledge and estimate future parking space availability based on workers' knowledge using locationdependent POI questions. We also propose an incentivisation scheme to reward workers based on the reliability of contributed knowledge.

- We perform extensive simulations to evaluate the performance of ParkCrowd. Moreover, ParkCrowd mobile application has been developed to carry out real-world experiments. Both simulation and real-life results indicate that ParkCrowd performs well in identifying unreliable information, scoring future availability of parking spaces and rewarding workers based on their reliability and efforts level.

The remainder of this paper is organised as follows: Section II presents the related work. Section III gives an overview of the ParkCrowd and highlights the problems the system aims to solve. The models used to evaluate the reliability of the workers' contributed knowledge, estimate the parking space availability and calculate rewards are discussed in Section IV.
Evaluation results are presented in Section V, including the simulation as well as the mobile system based real-world experiments. We conclude this paper in Section VI.

\section{RELATED WORK}

\section{A. Smart Parking}

Infrastructure based solutions using sensors or cameras have been proposed to measure parking occupancy [3], [14], [15], [16], [17]. A commercial product using sensors to detect vacancy of on-street parking spaces has been built [3]. [14] employs a multi-class SVM classifier to identify the state of parking spaces using images captured by security cameras. A method combining inter-space correlation and SVM using camera images is employed in [15]. However, placing dedicated sensors supposes either regular data collection by an employee, or wireless access, the approach also suggests either battery or dedicated power lines per unit. Using a camera sensing approach involves similar problems (internet connectivity, power supply) in addition to the problems of camera installation alignment, adjusting and positioning, and merging images from multiple cameras. Each parking lot has distinct features (like streetlight placement) that occlude the view from any single camera unit. This ensures that each camera deployment must be custom-tailored to each particular lot, increasing averaged system cost. In addition to these problems, for each deployment the detection algorithms used must be operator tuned to take shading and discolouration into account daytime, at night, and at all times in between these extremes. A more flexible approach is introduced to map parking spaces using vehicles' pre-installed ultrasonic parking sensors [13], which essentially builds a vehicle based crowdsensing system. However, the approach requires specific hardware configuration and thus can't be used in low-end or old vehicles. In addition, it is a vehicle-based rather than a phone-based parking search solution, thus it is less applicable when planning outside a vehicle.

Ubiquitous mobile crowdsensing (MCS) solutions by designing and implementing a smartphone application have been proposed as well. For example, smartphone applications such as Parker [3] and ParkSense [12] have been developed to allow users to view where parking spaces are located and also pay for them. However, there are significant limitations of these MCS based approaches. For example, the sensors are only able to detect the existence of parking spaces, but not other important information such as parking time, hourly price and whether it is private or public. Furthermore, these approaches are only able to detect real-time availability information and thus hindering the the drivers from planning their trip in advance based on future availability.

\section{B. Mobile Crowdsensing and Incentivisation}

Mobile Crowdsensing (MCS) paradigm aims to address the key challenges for data collection or truth discovery in the mobile environment by applying the "Human as a Service" [9], [18], [19] concept. It actively engages mobile users to perform more various types of data collection, knowledge discovery or even resource sharing tasks, such as collecting 
sensing data or content rich data e.g., pictures [20], [21], seeking knowledge from the crowd [22], [23], and performing location-dependent or time sensitive tasks [24], [25]. Due to the uncertainty in the performance of the crowd workers, MCS usually asks a number of workers to perform the same task, thus effectively discovering the truth based on crowd knowledge. Unfortunately, it is not always obvious how best to combine the crowds (workers), because the (often anonymous) workers have unknown and diverse levels of expertise, and the potential for systematic biases across the crowd exists. Naïve consensus methods which simply take uniform averages or the majority answer of the workers have been known to perform poorly [22]. Recently, some probabilistic and learning based methods have been proposed [26], [27], [28], [29]. For example, approaches using Expectation Maximisation (EM) have been employed to discover ground truth for events or other discrete variables [26], [27], [28]. However, such methods cannot be directly employed here since EM usually assumes a prior probability distribution for the events or discrete variables and it is infeasible to model the parking space availability as such. Unsupervised learning approaches are also used to perform truth discovery for sensing data [29]. However, the proposed approach is not able to capture any potential bias of the workers and thus requires much more data to achieve decent performance, which is not realistic in the real-time information collection for parking spaces.

Another important issue for MCS systems is incentivisation. Human are selfish and incentivisation schemes are essential to recruit and retain crowd workers to contribute their resources for computation and communication. Various schemes can be used for MCS systems. Most of the research has been focused on auction based methods to reveal the truthful cost of the participants when performing crowdsensing tasks [30], [31], [32]. However, the auction based methods tend to solicit prime quality data from strong players, while the aim of MCS is to achieve more participation for performing simple sensor data collection tasks [33], [34]. Moreover, it does not justify the reward distribution on other aspects, such as data quality, expertise or efforts level of the crowd workers, because the negotiated price in the auction process is usually not directly related to the results of data collection process. Therefore, incentivisation schemes which distribute rewards based on individual contributions of the workers after the data has been collected have been proposed recently [26], [29], [35]. [26] employs an entropy based method to quantify the contributions of the workers while [29] constructs the reputation of the workers for reward distribution. Shapley value based method is also used for reward distribution.

\section{PReliminaries}

In this part, we first highlight the challenges in building ParkCrowd system for collecting and disseminating parking space information. We then give an overview of the ParkCrowd system by introducing its important components, workflow and data models.

\section{A. Problem Statement}

There are three important functionalities ParkCrowd aims to provide:

- Collecting and disseminating real-time parking space information.

- Estimating future availability level of parking spaces.

- Distributing rewards to crowd workers.

In the following, the challenges of providing these functionalities are discussed and the measures ParkCrowd takes to meet these challenges are briefly introduced.

1) Reliability of real-time parking information: The reliability of the real-time parking space information is related to the fact that the reported parking space is indeed available, e.g., not privately owned or not accessible (e.g., outside parking hours) at the moment. In ParkCrowd, we assume that all workers are cooperating and are not contributing false information intentionally. However, the reliability issue still exists due to the heterogeneous knowledge level of the workers. Since the parking space information is location-dependent, if the workers are not familiar with an area, they may fail to identify a private parking space and treat it as vacant. Such knowledge based reliability issues must be identified by ParkCrowd in order to disseminate reliable information to the drivers. In ParkCrowd, we propose to learn the reliability of the information crowd workers submit based on their knowledge about that area using a set of POI questions. For example, the more a worker is knowledgeable about an area, the more likely the contributed knowledge is reliable. ParkCrowd employs a likelihood maximisation approach to perform reliability estimation for the crowd knowledge based on the answers collected from the workers for the POI questions.

2) Estimation accuracy of future parking space availability: To provide more insights to the drivers, ParkCrowd needs to infer the future availability level of the vacant parking spaces. The approach employed by ParkCrowd is to rely on human knowledge to estimate the availability level. The crowd workers are asked to give a numerical score for the availability level. In ParkCrowd, we set the range of the score to be $(0,5)$. The numerical score can be defined as the expected percentage of the number of vacant parking spaces at a future time point, with 5 indicating most parking spaces are vacant and 0 meaning that most spots will be occupied. However, crowd workers may have different perceptions on the same availability level due to their individual expertise level or personal biases. Therefore, to combine the workers' knowledge for estimating future availability level more effectively, ParkCrowd uses a series of control questions to learn the parameters of the workers such as the biases and remove their effects in estimating the true availability level. More specifically, crowd workers are asked to score the popularity level of nearby POIs in a similar way as scoring the parking space availability. Although the availability data for parking spaces are more suitable as control questions, due to the limited availability of such data, we use popularity level data of POIs instead as a proxy by assuming that workers score human activities related scenarios in a similar manner, e.g., with similar biases. 


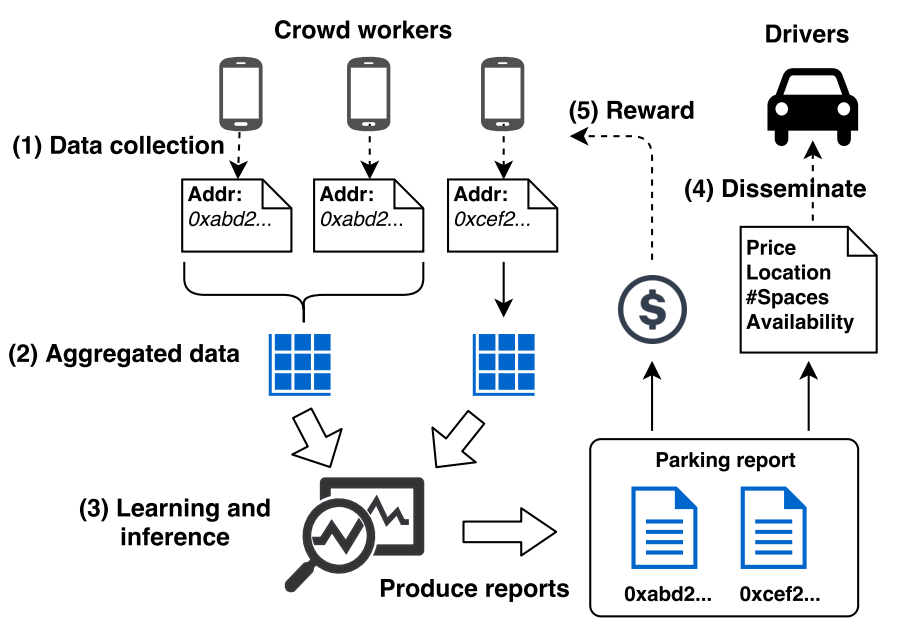

Fig. 2. Workflow of the ParkCrowd system.

3) Reward distribution based on knowledge reliability: Incentivisation scheme is important for recruiting, retaining and eliciting best quality of information collected from the crowd workers. Due to the importance of knowledge reliability for ParkCrowd, a reliability based reward distribution scheme is proposed. The reliability of crowd knowledge in ParkCrowd is affected by two factors. First, the workers' knowledge of the proximal area of parking spaces would lead to both unreliable real-time information and future availability estimation. Besides, the knowledge reliability for estimating future availability level is also determined by their efforts in scoring. If workers are careless in making predictions and give only a random score, the estimation accuracy will be adversely influenced. Therefore, ParkCrowd employs a reward distribution method by considering both factors of proximity based knowledge reliability and efforts level and make reliability related monetary payments to the workers.

\section{B. System Overview}

Fig. 2 gives an overview of ParkCrowd. In general, its workflow consists of five procedures:

1) Data collection: A number of crowd workers $\mathcal{W}=$ $\left\{w_{1}, w_{2}, \ldots, w_{M}\right\}$ use their mobile devices to upload data $\mathcal{D}=\left\{\mathcal{D}_{\text {hour }}, \mathcal{D}_{\text {price }}, \mathcal{D}_{\# \text { spots }}, \mathcal{D}_{\text {\#vacant }}, \mathcal{D}_{\text {avail }}\right\}$ for a group of parking spaces $\mathcal{S}_{\ell}$ at location $\ell$ to the cloud (ParkCrowd platform $\mathcal{P}$ ), where $\mathcal{D}_{\text {hour }}$ is time range data indicating parking hours, $\mathcal{D}_{\text {price }}$ is a decimal number for the (hourly) price of $\mathcal{S}_{\ell}, \mathcal{D}_{\# \text { spots }}$ and $\mathcal{D}_{\# \text { vacant }}$ is the number of all and vacant spots, and $\mathcal{D}_{\text {avail }} \in(0,5)$ shows the potential availability of $\mathcal{S}_{\ell}$ in the short term based on $\mathcal{W}$ 's knowledge, e.g., next 30 minutes, with 5 indicating the most of the parking spaces are very likely to be available and 0 indicating most spots will be occupied. The location $\ell$ for the group of parking spaces is indicated by $\mathcal{W}$ using the ParkCrowd mobile app in a way similar to input pick-up location on Uber app. Moreover, $\mathcal{W}$ need to answer a set of POI related control questions $\mathcal{Q}=\left\{q_{1}, q_{2}, \ldots, q_{N}\right\}$, which include the following two types of questions. An example of the collected answers for these two types of POI questions is shown in Fig. 3.

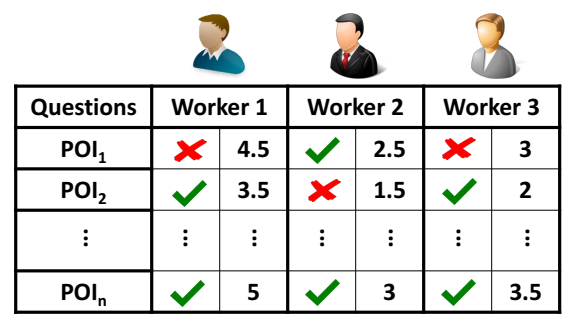

Fig. 3. Aggregated workers' answers for POI control questions.

- Proximity-based questions are used to identify the reliability of $\mathcal{W}$ 's knowledge for parking spaces at location $\ell$. When submitting information for $\mathcal{S}_{\ell}, \mathcal{W}$ are given a list of POIs and they need to indicate if a given POI is in proximity (e.g., $<100$ meters) of $\ell$ or not. The real labels for these questions $\boldsymbol{y}=\left\{y_{1}, \ldots, y_{N}\right\}$ are binary $\{0,1\}$, with 1 indicating a POI is close to $\ell$ and 0 otherwise. ParkCrowd use 30 questions $(N=30)$ and half of the questions are true (with label 1) and half are false (label 0). The workers' answers $\boldsymbol{X}=\left\{x_{i, j}\right\}$, where $i \in\{1, \ldots, N\}$ and $j \in\{1, \ldots, M\}$, are recorded as binary $\{-1,1\}$, with 1 indicating a POI for question $i$ is selected by worker $w_{j}$ as in proximity of $\ell$ and -1 otherwise. Such binary choices are shown in Fig. 3 as the $\boldsymbol{V}$ and $\boldsymbol{X}$. Based on these data, models can be built to identify unreliable information collected from $\mathcal{W}$.

- Human activity/popularity based questions are used to learn the parameters of $\mathcal{W}$, e.g., bias and variability, when they are estimating human activity level related situations, such as the availability of parking spaces, the popularity of a POI, etc. By combining the learned parameters of $\mathcal{W}$ from the answers and the collected parking space availability data $\mathcal{D}_{\text {avail }}$, the potential availability level $\hat{\mathcal{D}}_{\text {avail }}$ can be more accurately captured by removing the knowledge biases of $\mathcal{W}$. Although the best way to obtain such information is by collecting $\mathcal{W}$ 's knowledge on the availability level of parking spaces which have usage records, due to the limited availability of such information and the location-dependent nature of the system, we use the human activity level of nearby POIs as a proxy to learn the workers' parameters in ParkCrowd. The activity levels $\boldsymbol{Z}=\left\{z_{i, j}\right\}$, where $i \in\{1, \ldots, N\}$ and $j \in\{1, \ldots, M\}$, are scored by worker $w_{j}$ for POI question $q_{i}$ and $z_{i, j} \in(0,5)$, which has the same range as $\mathcal{D}_{\text {avail }}$. An example of the reported availability score is shown in Fig. 3.

2) Data aggregation: After $\mathcal{P}$ receives $\mathcal{D}$ and $\mathcal{Q}$, it aggregates them per location $\ell$ as $\mathcal{D}_{\ell}$ and $\mathcal{Q}_{\ell}$ based on the hash of the geo-decoded street address $h(g(\ell))$, where $h$ is a hash function and $g$ is geo-decoding function to obtain street address from GPS coordinates. Intuitively parking spaces $\mathcal{S}$ at different locations $\ell$ should be grouped based on the proximity of their GPS coordinates. However, street address based aggregation is used by ParkCrowd because $\mathcal{S}$ at the same street address would be more likely in the visual range of the drivers as a result of urban planning, thus avoiding the situation that $\mathcal{S}$ on the other side of a city block or a wide street but close in terms of GPS coordinates cannot be easily 
found by the driver. The answers for POI questions are also grouped per $\ell$ and processed by the system to identify workers' reliability and estimating parking space availability level based on workers' knowledge.

3) Data processing: There are two stages for the data processing. The first stage estimates the reliability of the realtime availability information collected by $\mathcal{W}$. There are two purposes of evaluating reliability: a) It captures the fact that the workers who are not familiar with the area are more likely to submit incorrect data, such as reporting private parking spaces, wrong parking price, etc. b) The familiarity of $\mathcal{W}$ affects the estimation accuracy of the future availability. The POIs in the questions are mostly nearby and the results are based on the workers' knowledge of those POIs. After the first stage, if the reliability of the information collected by a group of $\mathcal{W}$ is satisfactory, the data $\mathcal{D}_{\text {avail }}$ collected from the workers are then combined to learn the parameters of the workers and estimate the parking spaces' future availability level. Otherwise if the reliability is low, the collected information will not be processed further and disseminated by ParkCrowd. In the second stage, the diverse levels of expertise of the workers are learned first. The estimated future availability is computed by combining the crowd knowledge after eliminating the effects of diversity of expertise such as bias.

4) Data dissemination: Basic information of the parking spaces and the processed data, including the estimation results for future availability, are disseminated by $\mathcal{P}$ to the mobile applications installed on drivers' smartphones. As for the other types of data such as parking hours and hourly price, ParkCrowd adopts a recommender system style approach to show the drivers a list of the workers' answers ranked by their expertise level. ParkCrowd also implements a map view to show all nearby published parking space information on the mobile app.

5) Rewarding: Crowd workers that contribute information will receive certain amount of monetary payments from the platform $\mathcal{P}$. These reward payments come from the revenue of operating ParkCrowd system. For example, subscription fees that are paid by drivers can generate revenue for the system operator, while advertisement on the smartphone application is another potential revenue source. Moreover, to incentivise participation of $\mathcal{W}$ and encourage them to submit more reliable data, ParkCrowd applies a knowledge reliability based incentivisation scheme to reward crowd workers. The amount of the rewards $\mathcal{R}=\left\{r_{1}, \ldots, r_{M}\right\}$ for each worker $w_{j}$ are computed based on both the learned reliability, and their consistency in estimating the popularity levels of POIs, which reflect workers' efforts in estimating parking space availability level.

\section{MODELS}

This section presents the models used for estimating information reliability and parking space availability level based on crowd workers' knowledge. The reliability based incentivisation scheme is also discussed. As aforementioned, ParkCrowd uses location-dependent control data (POI questions) for both the reliability and availability estimation. The control data for reliability estimation are binary POI questions to test their familiarity with that area. The availability control data are human activity level score which can be used to learn the parameters for workers, such as their biases in making estimations.

\section{A. Reliability Estimation for Real-Time Information}

As introduced in Section III, $\mathcal{W}$ collect the answers $\boldsymbol{X}=$ $\left\{x_{i, j}\right\}$, where $i \in\{1, \ldots, N\}$ and $j \in\{1, \ldots, M\}$, for the true label $\boldsymbol{y}=\left\{y_{1}, \ldots, y_{N}\right\} . x_{i, j} \in\{-1,1\}$ and $y_{i} \in\{0,1\}$. Therefore, we model such problem of maximising the likelihood of the reliability of the information as a two class probabilistic classification problem. The two classes correspond to that the indicated free parking space is indeed available and accessible, for example, it is not private parking space, limited usage by member or electric car only, etc. The model aims to map the answers from crowd workers to a probabilistic judgement on the reliability of that parking space information. When workers submit answers $x_{i, j}$ for POI question $q_{i}$, the weights $w_{j}$ of the answers from each crowd worker $j$ can be learned from labels $y_{i}$. The training dataset is thus $\left(\boldsymbol{x}_{1}, y_{1}\right),\left(\boldsymbol{x}_{2}, y_{2}\right), \ldots,\left(\boldsymbol{x}_{N}, y_{N}\right)$, in which $\boldsymbol{x}_{i}=\left\{x_{i, 1}, \ldots, x_{i, M}\right\}$ is the input data for $q_{i}$ from all workers and $\boldsymbol{y}$ is the label vector. The relationship between $\boldsymbol{y}$ and $\boldsymbol{X}$ would be to maximise the likelihood of the following function:

$$
P(\boldsymbol{y} \mid \boldsymbol{\beta}, \boldsymbol{X})=\prod_{i=1}^{N} P_{i}\left(1 \mid \boldsymbol{\beta}, \boldsymbol{x}_{i}\right)^{y_{i}}\left(1-P_{i}\left(1 \mid \boldsymbol{\beta}, \boldsymbol{x}_{i}\right)^{1-y_{i}}\right),
$$

where $P_{i}\left(1 \mid \boldsymbol{\beta}, \boldsymbol{x}_{j}\right)=1 /\left(1+e^{-\boldsymbol{\beta}^{T} \boldsymbol{x}_{i}}\right)$ and $\boldsymbol{\beta}$ is the coefficients vector for the workers' answers $\boldsymbol{X}$ in the likelihood estimator. However, there are a limited number of POI questions that a worker can answer due to time limit and user experience. Moreover, the number of workers that contribute information for a specific parking space within a short time frame would also not be very large, e.g., up to 10 or 20 workers. Therefore, the number of parameters that need to be estimated is relatively large compared to the number of training data, which could cause overfitting problems. To mitigate such problem, we add a $\mathrm{L} 2$ regularisation term to the likelihood estimator:

$$
\begin{aligned}
\mathcal{L}(\boldsymbol{y}, \boldsymbol{X}, \boldsymbol{\beta}) & =-\ln P(\boldsymbol{y} \mid \boldsymbol{\beta}, \boldsymbol{X})+\frac{\lambda}{2}\|\boldsymbol{\beta}\|^{2} \\
& =\sum_{i=1}^{N}\left(1-y_{i}\right) \boldsymbol{\beta}^{T} \boldsymbol{x}_{i}+\ln \left(1+e^{-\boldsymbol{\beta}^{T} \boldsymbol{x}_{i}}\right)+\frac{\lambda}{2}\|\boldsymbol{\beta}\|^{2},
\end{aligned}
$$

To solve (2), one can compute the gradient descent w.r.t. $\boldsymbol{w}$ and find the optimal $\boldsymbol{w}$ step-by-step using the following formula:

$$
\boldsymbol{\beta}^{(k+1)}=\boldsymbol{\beta}^{(k)}+\eta \nabla_{\boldsymbol{\beta}} \mathcal{L}(\boldsymbol{y}, \boldsymbol{X}, \boldsymbol{\beta}),
$$

where

$$
\nabla_{\boldsymbol{\beta}} \mathcal{L}(\boldsymbol{y}, \boldsymbol{X}, \boldsymbol{\beta})=\sum_{i=1}^{n}\left(\frac{1}{1+e^{-\boldsymbol{\beta}^{T} \boldsymbol{x}_{i}}}-y_{i}\right) \boldsymbol{x}_{i}+\lambda \boldsymbol{\beta},
$$

The estimated likelihood of the reliability of the collected information can be made using $P\left(1 \mid \boldsymbol{x}^{\prime}, \boldsymbol{\beta}\right)$ where $\boldsymbol{x}^{\prime}$ is a vector with all elements being 1 indicating that all workers tend to declare the existence of the parking space. 


\section{B. Joint Estimator for Scoring Parking Space Availability}

To estimate the parking space availability from workers' provided score, one needs to know and remove the effects due to heterogeneous parameters of the workers such as their bias in making estimations in order to combine their answers more effectively. One way to address this problem is to score workers using their past performance on problems similar to the current problem. However, this is not always practical, since it is difficult and costly for anonymous workers to maintain historical records. Another problem with this approach is that worker's past tasks may be very different from the current task. One alternative approach is to "seed" some control questions with known answers into each worker's assigned tasks, then score each worker's reliability using these control questions, and weight their answers according to their reliability [36]. In ParkCrowd, we consider using a Gaussian model for modelling workers' parameters and the availability estimation:

$$
z_{i j}=\mu_{i}^{*}+b_{j}^{*}+\sigma_{j}^{*} \xi_{i j}, \quad \xi_{i j} \sim \mathcal{N}(0,1),
$$

where $\mu_{i}^{*}$ is the true answer for the question, and $b_{j}$ and $\sigma_{j}^{*}$ are bias and variability for the answer given by worker $j$. In the following, we will explain the model using control questions to improve the estimation of parking space availability information.

Joint Estimator: we directly maximise the joint likelihood of the crowdsensed availability data $\left\{z_{i j}\right\}$ of both target and control questions, with $\mu_{\mathcal{C}}$ of the control questions clamped to the true values $\mu_{\mathcal{C}}^{*}$. That is,

$$
\begin{aligned}
{\left[\hat{\mu}_{\mathcal{T}}, \hat{b}, \hat{\sigma}\right]=} & \underset{\left[\mu_{\mathcal{T}}, b, \sigma\right]}{\arg \max }\left\{\sum_{i \in \mathcal{C}} \sum_{j \in \partial_{i}} \log p\left(z_{i j} \mid \mu_{i}^{*}, b_{j}, \sigma_{j}\right)\right. \\
& \left.+\sum_{i \in \mathcal{T}} \sum_{j \in \partial_{i}} \log p\left(z_{i j} \mid \mu_{i}, b_{j}, \sigma_{j}\right)\right\}, \\
= & \underset{\left[\mu_{\mathcal{T}}, b, \sigma\right]}{\arg \max } f(\boldsymbol{Z}, \boldsymbol{\mu}, \boldsymbol{b}, \boldsymbol{\sigma}),
\end{aligned}
$$

where $\boldsymbol{Z}=\left\{z_{i, j}\right\}$ represents the matrix storing the label for question $q_{i}$ submitted by worker $j, \boldsymbol{\mu}=\left\{\mu_{i}\right\}$ is the vector of true label for all questions, $\boldsymbol{b}=\left\{b_{j}\right\}$ and $\boldsymbol{\sigma}=\left\{\sigma_{j}\right\}$ are the vectors of the biases and variabilities of all workers respectively, $\mathcal{C}$ is the set of control questions, $\mathcal{T}$ is the set of target questions the model aims to estimate, and $\partial_{i}$ is the set of workers who submit answer for $q_{i}$. Therefore,

$$
\begin{aligned}
f(\boldsymbol{Z}, \boldsymbol{\mu}, \boldsymbol{b}, \boldsymbol{\sigma})= & \sum_{i \in \mathcal{C}} \sum_{j \in \partial_{i}} \log p\left(z_{i j} \mid \mu_{i}^{*}, b_{j}, \sigma_{j}\right) \\
& +\sum_{i \in \mathcal{T}} \sum_{j \in \partial_{i}} \log p\left(z_{i j} \mid \mu_{i}, b_{j}, \sigma_{j}\right) \\
= & -\left\{\sum_{i \in \mathcal{C}} \sum_{j \in \partial_{i}} \frac{\left(z_{i j}-b_{j}-\mu_{i}^{*}\right)^{2}}{2 \sigma_{j}^{2}}+\log \sigma_{j}\right. \\
& \left.+\sum_{i \in \mathcal{T}} \sum_{j \in \partial_{i}} \frac{\left(z_{i j}-b_{j}-\mu_{i}\right)^{2}}{2 \sigma_{j}^{2}}+\log \sigma_{j}+C\right\},
\end{aligned}
$$

where $C=\left\{\sum_{i \in \mathcal{C}} \sum_{j \in \partial_{i}} \frac{1}{2} \log 2 \pi+\sum_{i \in \mathcal{T}} \sum_{j \in \partial_{i}} \frac{1}{2} \log 2 \pi\right.$.
Suppose there are $M$ crowd workers and $N$ data, $\hat{b}_{j}, \hat{\sigma}_{j}$ and $\hat{\mu}_{i}$ can be computed using Block Coordinate Descent (BCD), which alternatively updates the variables till convergence by computing the gradient decent of the joint estimator's objective function in (6). For the sequence of updated variables $\hat{\boldsymbol{b}}^{k}=$ $\left\{\hat{b}_{1}^{k}, \ldots, \hat{b}_{M}^{k}\right\}$ and $\hat{\boldsymbol{\mu}}_{k}=\left\{\hat{\mu}_{1}^{k}, \ldots, \hat{\mu}_{N}^{k}\right\}$ at the $k$-th iteration of $\mathrm{BCD}$, the following calculation are performed:

$$
\begin{gathered}
\frac{\partial f(\boldsymbol{Z}, \boldsymbol{\mu}, \boldsymbol{b}, \boldsymbol{\sigma})}{\partial b_{j}}=\sum_{i \in \partial_{j}^{c}} \frac{z_{i j}-b_{j}-\mu_{i}^{*}}{\sigma_{j}^{2}}+\frac{z_{i j}-b_{j}-\mu_{i}}{\sigma_{j}^{2}}, \\
\frac{\partial f(\boldsymbol{Z}, \boldsymbol{\mu}, \boldsymbol{b}, \boldsymbol{\sigma})}{\partial \mu_{i}}=\sum_{j \in \partial_{i}} \frac{z_{i j}-b_{j}-\mu_{i}}{\sigma_{j}^{2}},
\end{gathered}
$$

where $\partial_{j}^{\mathcal{C}}$ and $\partial_{j}^{\mathcal{T}}$ are the answers given by worker $j$ for the questions in the control set $\mathcal{C}$ and in the target set $\mathcal{T}$ respectively. Therefore, given $\hat{\boldsymbol{\mu}}^{k}$, one can find the next $\hat{\boldsymbol{b}}^{k+1}$ and $\hat{\boldsymbol{\mu}}^{k+1}$ by:

$$
\begin{gathered}
\hat{b}_{j}^{k+1}=\frac{\sum_{i \in \partial_{j}^{\mathcal{C}}}\left(z_{i, j}-\mu_{i}^{*}\right)+\sum_{i \in \partial_{j}^{\mathcal{T}}}\left(z_{i, j}-\hat{b}_{j}^{k}\right)}{\left|\partial_{i}\right|}, \\
\hat{\mu}_{i}^{k+1}=\sum_{j \in \partial_{i}}\left(z_{i, j}-\hat{b}_{i}^{k+1}\right),
\end{gathered}
$$

Moreover, to better differentiate the performance of the workers, we also compute the weights for each worker after obtaining $\hat{\boldsymbol{b}}_{k+1}$ when estimating $\hat{\boldsymbol{\mu}}_{k+1}$ :

$$
\hat{w}_{j}=\left|\partial_{j}\right| /\left(\sum_{i \in \partial_{j}}\left(z_{i j}-\hat{\mu}_{i}^{k}-\hat{b}_{j}^{k+1}\right)^{2}\right),
$$

where $\partial_{j}=\partial_{j}^{\mathcal{C}} \cup \partial_{j}^{\mathcal{T}}$. The estimated true value at $(\mathrm{k}+1)$-th iteration is:

$$
\hat{\mu}_{i}^{k+1}=\sum_{j \in \partial_{i}} \hat{w}_{j}\left(z_{i j}-\hat{b}_{j}^{k+1}\right) / \sum_{j \in \partial_{i}} \hat{w}_{j},
$$

\section{Reliability Based Incentivisation Scheme}

The incentivisation scheme for ParkCrowd is trying to capture the heterogeneous reliability of workers' knowledge in collecting parking space information. As aforementioned, the scheme focuses on the reliability of location-dependent knowledge and the efforts level of workers in estimating parking space availability level. In general, to calculate the amount of reward, ParkCrowd takes two steps:

1) The coefficient $\boldsymbol{\beta}$ obtained from reliability estimation model is used to eliminate unreliable workers in the rewarding process.

2) The efforts level of the remaining workers are decided based on their variability in estimating the human activity level related questions including POI popularity and parking space availability.

The first step employs the POI questions to identify outliers, i.e., workers not familiar with the area. We treat those who have low reliability level as outliers and directly exclude them from receiving rewards. The main objective of the second step is to identify the workers who are careless in making estimations, which reflects the efforts level of the workers. Moreover, the second step can give further hints on 
the reliability of workers' knowledge. One can identify the different efforts levels by computing the variability $\hat{\sigma}_{j}$ of the worker $j$ :

$$
\hat{\sigma}_{j}=\sqrt{\sum_{i=1}^{N}\left(z_{i, j}-\mu_{i}^{*}-\hat{b}_{j}\right)^{2} / N},
$$

The following theorem indicates that the workers' variability has direct impact on the estimation of the parking space availability in terms of the mean squared error (MSE) of the joint estimator.

Theorem 4.1: Assume the crowdsensed data $\left\{z_{i j}\right\}$ are drawn from $p\left(z_{i j} \mid \mu_{i}^{*}, b_{j}^{*}, \sigma_{j}^{*}\right)$, where $\left\{\mu_{i}^{*}\right\}$ and $\left\{b_{j}^{*}\right\}$ are drawn from priors $Q_{\mu}$ and $Q_{b}$ respectively. Asymptotic expected MSE of the joint estimator defined in Subsection IV-B is:

$$
\mathbb{E}\left[\left\|\hat{\mu}-\mu^{*}\right\|^{2}\right] \approx \mathbb{E}\left[\operatorname{tr}\left(\left(\boldsymbol{H}_{\boldsymbol{\mu} \boldsymbol{\mu}}-\boldsymbol{H}_{\boldsymbol{\mu} \boldsymbol{\nu}} \boldsymbol{H}_{\boldsymbol{\nu} \boldsymbol{\nu}}^{-1} \boldsymbol{H}_{\boldsymbol{\mu \nu}}^{T}\right)^{-1}\right)\right],
$$

where $\boldsymbol{H}_{\boldsymbol{\mu} \boldsymbol{\mu}}=R_{t} \otimes \mathbb{E}_{\mu}\left(H_{\mu \mu}\right), \boldsymbol{H}_{\boldsymbol{\nu} \boldsymbol{\nu}}=\left(L_{t}+L_{c}\right) \otimes \mathbb{E}_{\mu}\left(H_{\nu \nu}\right)$, where $H_{\mu \mu}=-\mathbb{E}_{z}\left[\nabla_{\mu \mu}^{2} \log p(z \mid \mu, b, \sigma)\right]$ is the Fisher information matrix (similarly for $H_{\nu \nu}$ ), $\otimes$ is the Kronecker notation. If all questions are answered by all workers, $L_{t}+$ $L_{c}=N I, R_{t}=M I, N$ is the number of POI questions, $M$ is the number of workers, and $I$ is the identify matrix. $\boldsymbol{H}_{\boldsymbol{\mu \nu}}=\left[H_{\mu_{i} \nu_{j}}\right]_{i, j}$ is a block matrix, where block $H_{\mu_{i} \nu_{j}}=$ $-\nabla_{\mu \nu}^{2} \log p(z \mid \mu, b, \sigma)$ with a random copy of $z, \mu, b$ and $\sigma$.

We want to reward workers based on their efforts in obtaining the asymptotic estimation given by (15), however, the contributions for individual workers are difficult to quantify and compare using (15), especially when the number of answers given by the workers are different. We propose the following simplified version to quantify individual efforts level for reward distribution by focusing only on $\operatorname{tr}\left(\left(H_{\mu \mu}\right)^{-1}\right)$. It can also be shown that $\operatorname{tr}\left(\left(H_{\mu \mu}\right)^{-1}\right)$ in most cases is also a good indicator for the MSE for the estimator [37], [38]. Given:

$$
\operatorname{tr}\left(\left(H_{\mu \mu}^{-1}\right)\right)=\frac{1}{\sum_{j=1}^{M} \frac{1}{\hat{\sigma}_{j}^{2}}},
$$

By combining the results of $\boldsymbol{\beta}$ and $\left.\operatorname{tr}\left(H_{\mu \mu}\right)^{-1}\right)$, the final reward is calculated as the percentage of the individual worker's effort level over all efforts in minimising the estimation error:

$$
r_{j}= \begin{cases}\frac{\frac{1}{\hat{\sigma}_{j}^{2}}}{\sum_{j=1}^{M} \frac{1}{\hat{\sigma}_{j}^{2}}} \mathcal{R}, & \text { if } \beta_{j}>t h, \\ 0, & \text { otherwise, }\end{cases}
$$

where th is a threshold for eliminating unreliable workers using proximity based POI questions, $\mathcal{R}$ is the total revenue to be distributed among the workers and $\hat{\sigma}_{j}^{2}$ are the estimated variability of the workers in answering human activity related questions.

\section{Summary}

Overall, the operations ParkCrowd performs are summarised in Algorithm 1. In terms of the complexity of the operations, every iteration in step 1 and step 2 both takes $\mathcal{O}(M N)$ and the computation of rewards takes $\mathcal{O}(M)$. In practice, the convergence speed of the operations in step 1 and 2 are

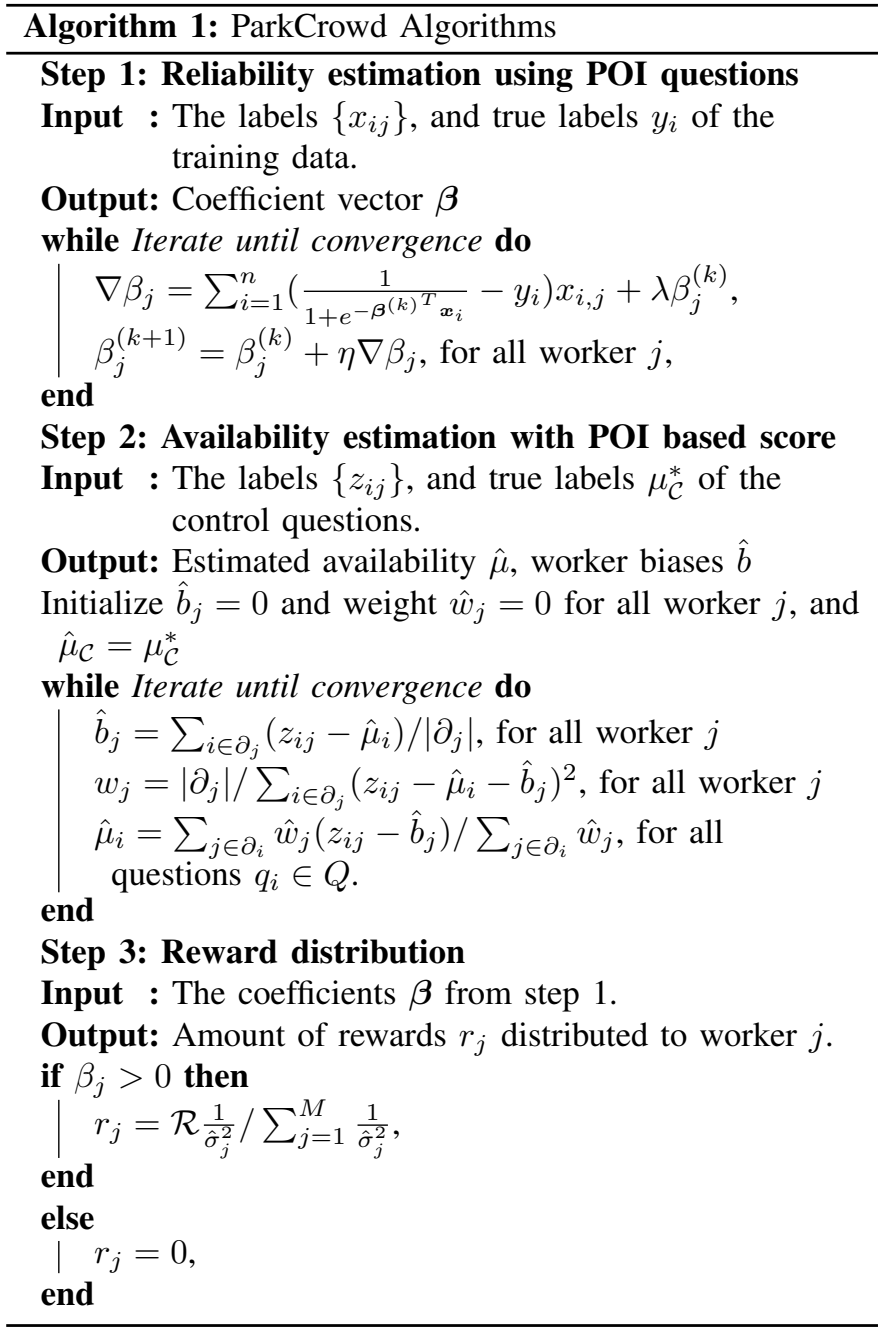

usually very fast due to the limited number of workers and control questions. Therefore, the computational time is not a big concern for the models.

\section{Evaluation}

We evaluate the performance of ParkCrowd using both reallife experiments and simulations. In the real-world experiments, we recruit workers to report parking space data using the ParkCrowd mobile application we developed for Android systems. A back-end cloud service is also implemented for data aggregation, storage and processing. The experiment results indicate that ParkCrowd can successfully identify unreliable information, make accurate estimation on future availability, capture the expertise levels of crowd workers and reward them accordingly. To evaluate the performance of the system on a large scale, we also perform extensive simulations.

\section{A. Prototype Implementation and Experiments}

We implement the ParkCrowd mobile application (mobile app) on Android OS and the back-end cloud server using nodejs. The communication between the mobile app and cloud server is conducted via several Rest APIs [39]. The user interface (UI) of the mobile application is shown in Fig. 4. 


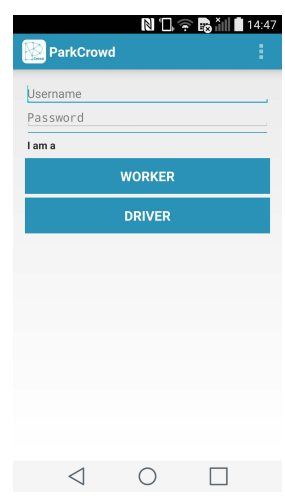

(a) Login

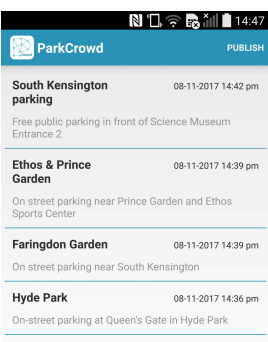

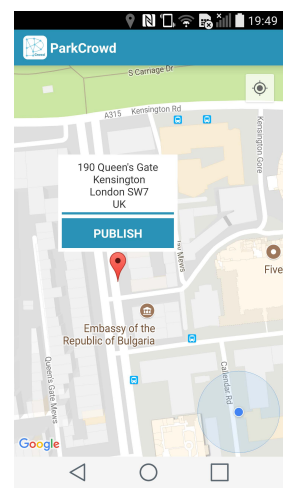

(c) Locate spaces

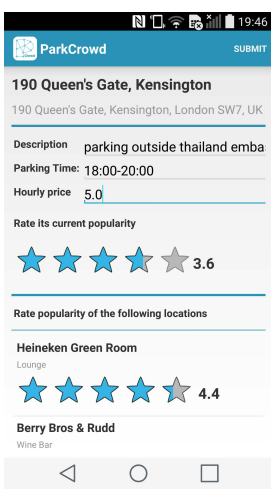

(d) Publish and answer POI questions

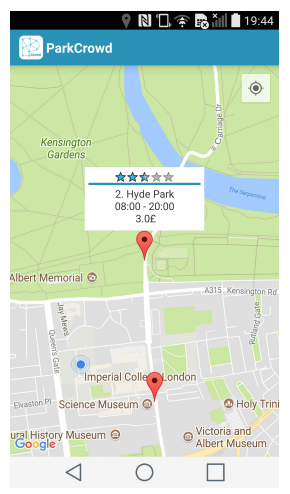

(e) Parking map

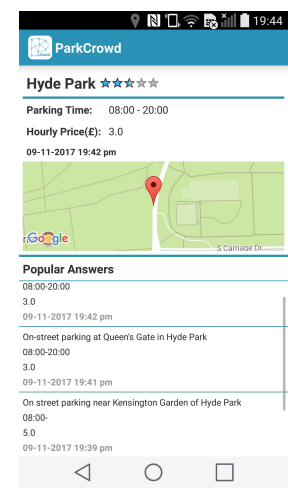

(f) Parking space details

Fig. 4. UI of ParkCrowd application.

The app can differentiate the roles of crowd workers and drivers using the login page shown in Fig. 4(a). The crowd workers are able to view the list of previously reported parking space information (Fig. 4(b)), select the location of the vacant parking spaces they want to report using a Google Map based service (Fig. 4(c)), and submit relevant information and answer POI questions (Fig. 4(d)). After receiving the reported information, the cloud server aggregates and processes the data and disseminates the results to the drivers, who are able to view all recently reported vacant parking spaces on a map (Fig. 4(e)) and check the details of them (Fig. 4(f)) using the mobile app.

1) Experiment settings: We have carried out real-life experiments using the developed ParkCrowd prototype to crowdsense parking space information at seven different locations in London. The full list of locations is shown in Table I. All locations provide on-street public parking spaces. For each experiment, 10 workers (college students and researchers) are recruited to report the parking space information and answer POI related questions. Moreover, at each location, a certain number of recruited workers are local, which means that the locations of the parking spaces are in proximity of some places they are very familiar with, such as their homes or workplaces. In terms of the evaluation metrics, we focus on 1) the accuracy of the estimated availability of the parking spaces, and 2) the impact of workers' expertise on the overall reliability of information, and 3) the amount of rewards received. The time when the experiments are carried out is between 9:00-9:30 during the morning rush hour when ParkCrowd would be mostly used in the real world. The ground truth occupancy information of the parking spaces are also collected at the same time ${ }^{1}$. In particular, we record the workers' reported information and answers at 9:00 and the vacant parking space information at 9:30 respectively. The availability level of recorded parking space information is converted to a score between 0 and 5 using the following formula:

$$
\frac{\# \text { vacant spaces at 9:30 }}{\# \text { total spaces }} \times 5 \text {, }
$$

\footnotetext{
${ }^{1}$ With special thanks to Pamela Bezerra for collecting the data.
}

For example, for location \#2, there are 10 spaces in total and 8 of them are vacant at 9:30, then the availability score for the parking spaces at location $\# 2$ is $8 / 10 \times 5=4$.

Another important issue for the ParkCrowd system is how to obtain the ground truth data for popularity levels of POI, which are essential for learning the workers' parameters. In ParkCrowd, we collect the check-in data from FourSquare [40] and use the number of check-ins as the indication of popularity level, given the assumption that people estimate both the availability of parking spaces and popularity level of POI based on their perceptions on human activity level. To align the score of popularity level to that of parking space availability, we apply the following formula to the obtained check-ins counts to transform it to the range of $(0,5)$.

$$
\min \left(\log _{10} \text { (\# check-ins), } 5\right) \text {, }
$$

The reason we use a $\log _{10}$ function here is that the cumulative distribution function (CDF) of the check-in counts for the POI has a similar shape to $10^{x}$, thus a transformation using $\log _{10}$ gives a linear approximation. The min operation is mainly used to remove outliers and does not have big effects on the final score, which can be proved by the simulation results.

TABLE I

LIST OF PARKING LOCATIONS FOR REAL-LIFE EXPERIMENTS

\begin{tabular}{cccc}
\hline \hline ID & \#vacant/all spaces & \#local workers & Location \\
\hline \hline 1 & $10 / 30$ & 1 & Canada Place \\
\hline 2 & $8 / 10$ & 5 & Griffon House, Grant Road \\
\hline 3 & $8 / 20$ & 6 & 7 Imperial College Rd \\
\hline 4 & $5 / 10$ & 6 & 186 Queen's Gate \\
\hline 5 & $8 / 10$ & 2 & 213 Townmead Road \\
\hline 6 & $16 / 20$ & 5 & 20 Grant Road \\
\hline 7 & $3 / 10$ & 6 & 25 Exhibition Road \\
\hline \hline
\end{tabular}

2) Experiment results: Fig. 5 shows the results of 1) the estimated reliability of the collected parking space information and 2) the estimated availability score (0-5) at the seven locations. The reliability is shown as a probability, which is computed using the logistic function in Section IV. As illustrated according to the red line in Fig. 5, the information at locations with lowest number of local workers (i.e., location \#1) has lowest reliability probability, which indicates that the 


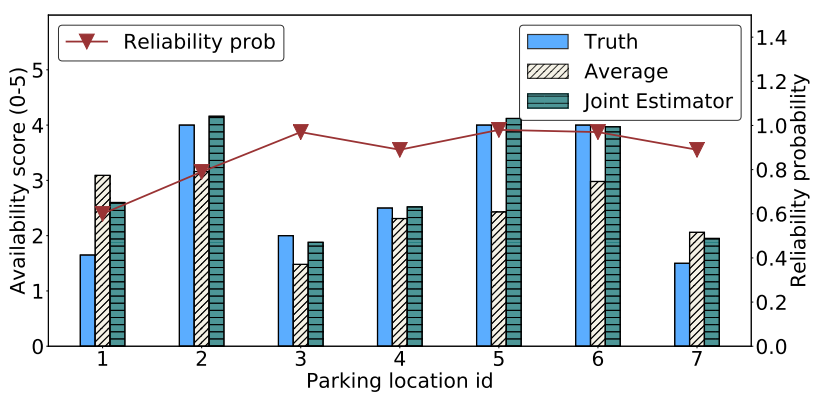

Fig. 5. Experiment results for information reliability and estimated availability (x-axis shows the ID of the locations as listed in Table. I).

system is less confident with the information obtained from the workers at these locations. Moreover, the proposed joint estimator also successfully removes the impact of workers' biases in their estimation. The estimation obtained using the joint estimator is more accurate in six of the seven locations and achieves an improvement of roughly 30\% compared to using the average availability score. Another observation is that when the reliability probability is higher, the estimation is also closer to the ground truth.

Fig. 6 shows the amount of rewards distributed to the workers (ranked by the amount). The threshold th used for eliminating workers in the reward distribution process is 0 . The red bars indicate the rewards for local workers, who are supposed to be more familiar with the areas and receive more rewards, and the blue bars indicate the rewards paid to random workers. Moreover, based on the reward distribution model shown in Subsection IV-C, some workers are eliminated from the reward distribution process and receive zero reward due to the low reliability. As illustrated in Fig. 6, the workers receiving the highest rewards are mostly local and familiar with the locations of the parking spaces. However, some local workers are also eliminated in the reward distribution process and receive zero reward. This could be due to the selected threshold. Take the location \#7 for example, if the threshold is reduced to -0.1 , both of the two local workers who are eliminated with $t h=0$ would get paid. Another phenomenon is that some workers who are not local still get paid. This can be explained from the following two perspectives: 1) In the control questions, the names and the types (e.g. pub/museum etc.) of the POIs are displayed on the application, which makes it possible for non-local workers to make reasonable guesses. For example, when a museum type POI is shown, non-local workers can guess a higher popularity score based on the fact that museums usually attract a large number of visitors; 2) When most workers are non-local, the model's parameters could be skewed in a favourable way for the random workers. Nevertheless, as for both cases, the rewards received by the random workers are still much less than the local ones. For example, as for location \# 1 (Canada Palace), though nine of the ten workers are random and five of them receive rewards, the amount they receive is extremely low compared to the single local worker.

\section{B. User Feedback and Discussion}

We collect some feedback from ParkCrowd users during the experiments. Based on their feedback, the current limitations and a number of possible improvement which could be made to ParkCrowd are discussed.

1) Data aggregation method: An important aspect mentioned by the users is the data aggregation method. Some users tend not to be able to accurately specify the locations of the parking spaces on Google Map, thus making the system fail to aggregate the information for the same group of parking spaces. Different users also tend to have different interpretations for the parking spaces at the same location. For example, some users treat $10-15$ parking spaces as the same location while others tend to group them with a smaller range or distance. However, once the basic information such as location and number of spots of on-street parking spaces can be collected on a large scale, ParkCrowd would be able to infer the grouping information in a better way to improve the current aggregation method, such as by employing a hybrid approach using both address, street map, distance, etc.

2) POI questions and scoring process: The POI and the parking space availability questions sometimes cause confusion to the users. The ParkCrowd UI adopts a rating based approach, however, such approach does not directly relate to any quantifiable way for parking space availability. Some users suggest using the number of vacant parking spaces for the input data. However, this suggestion may not work if the data aggregation method fails to differentiate the groups of parking spaces. One potential solution is to give more detailed instructions to the users to educate them about the idea behind the UI design and the purpose of the application as a whole. Although it is time consuming to educate new users to use the app in this way, most users tend to perform the tasks in a consistent way for the following experiments after the initial instructions.

3) Additional functionalities: Based on the feedback of ParkCrowd users, two additional functionalities are highly sought after. The first one is to offer in-app parking space reservation function. However, due to the public nature of the on-street parking and high cost for related infrastructures, it is unrealistic to provide any reservation service through ParkCrowd. Another suggested functionality which seems to be more feasible is route planning, which provides drivers guidance on how to plan their route to find vacant parking spaces based on the collected data and their journey destinations. This route planning problem can be solved using certain combinatorial optimisation frameworks such as maximum coverage or shortest path. However, due to the uncertainty for the availability estimation, a probabilistic approach would be more appropriate. We plan to address relevant issues in the future work.

\section{Simulations}

Since a large-scale real-life experiment is infeasible due to limitations of resources and manpower, we carry out simulations to examine the performance of ParkCrowd on a larger 

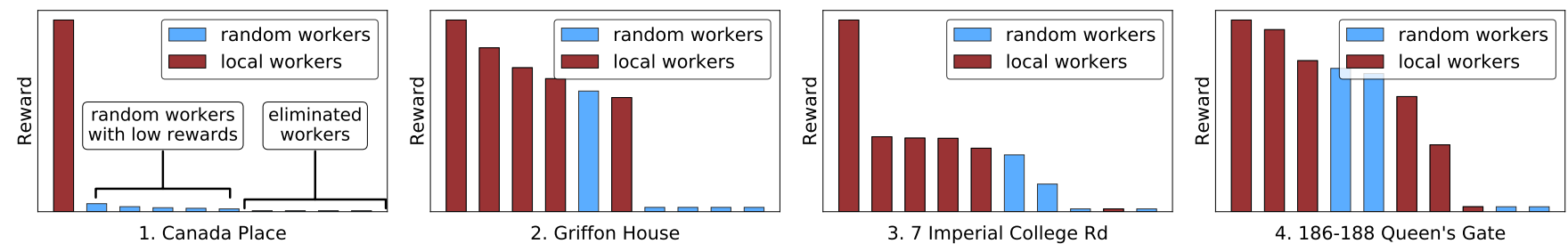

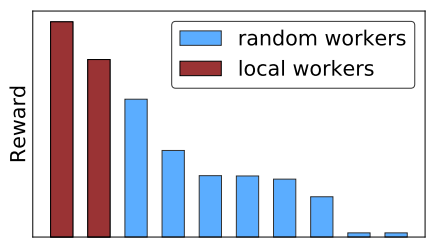

5. 213 Townmead Rd

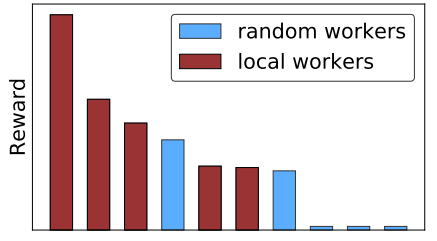

6. 20 Grant Rd

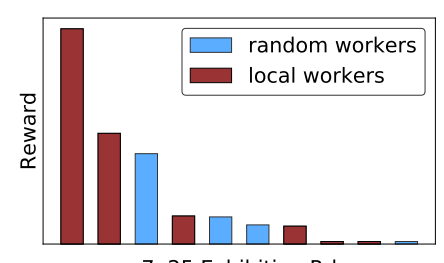

7. 25 Exhibition $\mathrm{Rd}$

Fig. 6. Rewards paid to workers at seven parking locations: 1) all workers (blue and red) are ranked by their obtained rewards; 2) local workers (red) receive more rewards than an average worker. The amount of rewards shown in the figure is normalised.

scale. Similar to real-world experiments, we evaluate the performance of estimating the information reliability, estimating future availability as well as distributing rewards.

1) Datasets: We collect one week parking space availability data of 95 parking lots in New York from [4]. Because the data of on-street and roadside parking spaces are difficult to obtain, we use parking lots data as proxy for the on-street and roadside parking space data in the simulations, which we believe would reflect the performance of ParkCrowd in a very similar way. For each parking lot, 10 workers are recruited to report information. We use 10 workers because we believe in real-life scenarios, there would not be a large number of workers reporting parking space information within a short time frame (e.g., 1-2 minutes) as we focus on collecting real-time information. The workers are split into local and random workers. However, although there are some parking spaces availability data, the workers' estimation data for the reliability and availability cannot be easily obtained. In order to mitigate the problem of missing data, we make some simple assumptions which are then used to generate crowdsensed data:

- Different types of workers have different probabilities to answer a question correctly. Therefore, we introduce an evaluation parameter for the probability that local workers give correct answers.

- The worker predicted availability data are generated using Gaussian models $\mathcal{N}\left(b_{j}+\mu_{i}, \sigma_{j}^{2}\right)$ as in (5). The bias $b_{j}$ and variability $\sigma_{j}^{2}$ of the workers are also chosen randomly in the simulations.

2) Evaluation parameters: The following two parameters are evaluated in the simulations:

- Percentage of local workers refers to the ratio of local workers among all workers for each simulation. For a specific parking lot in the simulations, if five of the ten workers are assumed to be local, the percentage of local workers is then $50 \%$. The simulations use a range between $0 \%$ (no local workers) and 100\% (all workers are local).

- Probability of correct answers from local workers indicates the chance that a local worker is able to answer a question correctly. It has different meanings for reliability and availability estimation. As for reliability, a probability of 0.9 indicates that a local worker is able to answer $90 \%$ of the binary POI questions correctly, while for availability estimation, it refers to the probability that the local worker will give a normally distributed score with the mean equal to his or her bias plus the ground truth popularity level. A range between $50 \%$ and $100 \%$ is used for this probability in the simulations.

The reason we choose these two parameters is that the percentage of local workers is very likely to affect both the estimated information reliability and availability. Intuitively, the larger percentage of local workers, the more reliable and accurate the crowdsensed information would be. The probability that a local worker gives a correct answer also influences the performance and it is meaningful to study the impact of this probability on the reliability and availability estimation performance of the proposed models. In the simulations, the probability that a random worker gives a correct answer is set to be 0.5 .

3) Results: For each combination of evaluation parameters and parking lot, 50 simulations are performed (overall 313500 simulations have been carried out). Fig. 7 shows the simulation results for reliability estimation. The system is able to give a high reliability (e.g., 0.9) if at least $20 \%$ workers are local and if they are able to give correct answers to POI questions with a probability of $90 \%$. This is an indicator for potential good performance since not so many local workers are required to achieve a confident level of the reliability of the information collected. Moreover, with more local workers reporting parking space information, the required probability of correct answers rapidly declines. For example, if all workers are local, a 0.6-0.7 probability of getting correct answers would be sufficient for the system to give a high reliability estimation (e.g., 0.9).

As for availability estimation results, Fig. 8 indicates strong performance. When the local workers account for $50 \%$ of all workers and can answer POI questions correctly with 0.8 probability, the estimation error is below 0.5 (of a score between 0 and 5). Moreover, with high percentage of local 


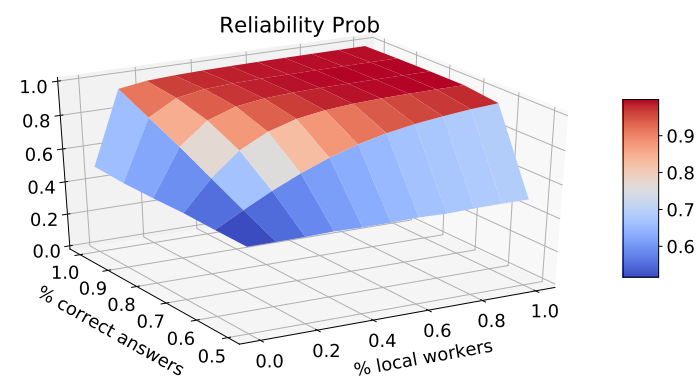

(a) Reliability probability 3D surface

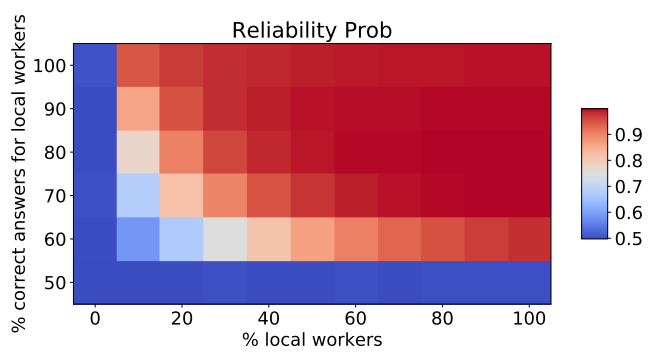

(b) Reliability probability $2 \mathrm{D}$ projection

Fig. 7. Simulated reliability probability (z-axis) w.r.t. 1) the percentage of local worker among all workers (x-axis) and 2) the probability local workers give correct answers (y-axis).

workers and high probability of correct answers, the error can be as low as 0.1. Compared to the percentage of local workers, the parameter for probability of getting correct answers plays a more important role. If the local workers are able to answer all questions correctly, only $10 \%$ of local workers is able to reduce the estimation error below 0.5 . Besides, the results indicate that although the model makes approximations by constraining the Gaussian score between 0 and 5 , it is still able to produce accurate estimation by capturing the bias and variability of the workers.

Fig. 9 shows the simulation results for reward distribution. Since 10 workers are used in each experiment, the average normalised reward for each worker is 0.1 . There are a number of key observations from Fig. 9. First, the surface in the left subfigure is a strict upper-bound for the surface on the right, which means that the reward received by a local worker is strictly higher than a random worker. Moreover, when the number of local workers is small (e.g., one local worker), the rewards received by local workers is much higher (close to 1 ) than random workers (close to 0 ). The only scenario that the rewards received by random worker is close to local workers is when local workers give random answers (\% 50 correct answers). In this case, both local and random workers will receive 0.1 on average.

\section{Discussion ANd CONCLUSIONS}

\section{A. Discussion}

We discuss this paper with a focus on its limitations and propose potential future work:

a) POI based control questions: This paper employs POI based control questions to reduce the estimation error of parking availability. However, POI data are not directly

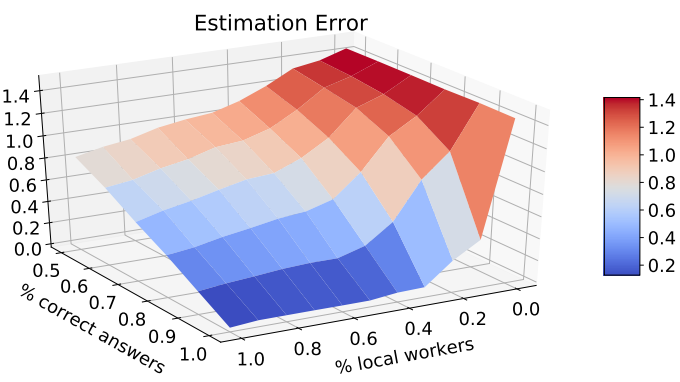

(a) Availability estimation error 3D surface

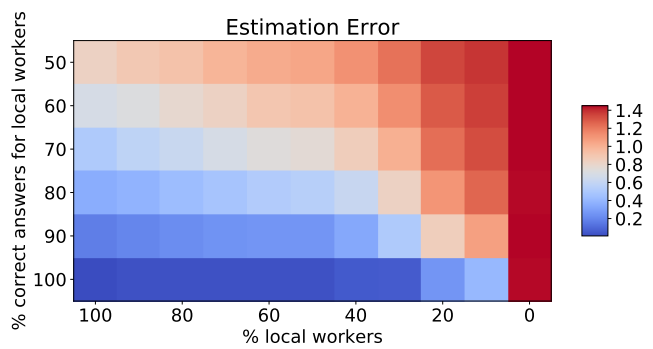

(b) Availability estimation error $2 \mathrm{D}$ projection

Fig. 8. Simulated errors of availability estimation (z-axis) w.r.t. 1) the percentage of local worker among all workers (x-axis) and 2 ) the probability local workers give correct answers (y-axis).

related to the parking data, which would make it difficult for the model to fully capture the perception errors of crowd workers. However, to our best knowledge, such data are not widely available. Therefore we choose to fuse FourSquare POI popularity data into the model to indirectly estimate the perception errors of crowd workers. In the future, we plan to collect and build our own datasets for parking spaces to improve the performance of the proposed methods.

b) Combined sensor and crowd based approach: This paper aims to build a cheap solution to report parking availability without relying on fixed infrastructure due to deployment cost. However, it would be interesting to build a hybrid approach that explores both sensor and crowd data. For instance, sensor nodes that are deployed on parking spaces can help profile the historical availability level of the parking spaces. Using both historical data and crowd contributed information may improve the accuracy of short-term predictions. We propose exploring this solution to find out the trade-offs in terms of cost and accuracy in future work.

\section{B. Conclusions}

In this paper, we propose ParkCrowd, which collects and disseminates parking space information based on crowd knowledge. ParkCrowd offers the drivers not only the basic information of parking spaces such as location, hourly price and real-time vacancy status, but also makes estimation of future availability of the parking spaces by aggregating crowd workers' knowledge. To estimate the reliability of the information reported by the crowd workers, we introduce locationdependent POI questions to dynamically score the workers' expertise. Two different probabilistic models have been proposed to infer the collective reliability of the information for vacant 

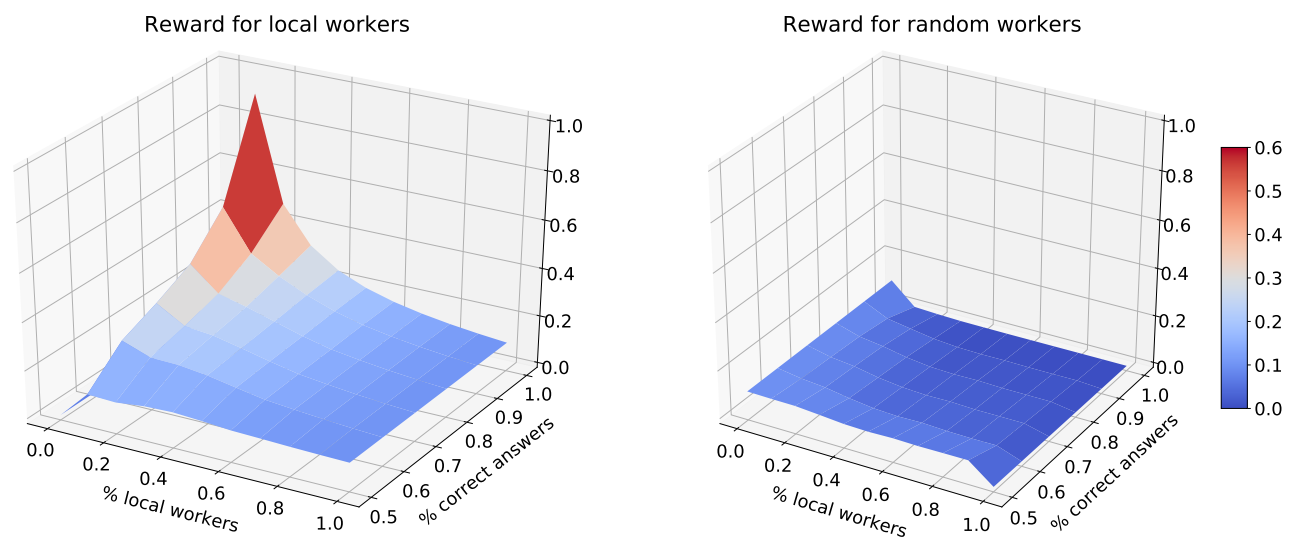

Fig. 9. Rewards paid to local workers (left) and rewards for random workers (right). The rewards received by local workers strictly dominate (as an upper bound) the rewards for random workers.

parking spaces as well as the accuracy of the estimation for the availability level. Both real-world experiments and simulations indicate strong performance in identifying unreliable crowd contributed information and making accurate estimation of parking space availability. Moreover, ParkCrowd also implements an incentivisation scheme to distribute rewards to crowd workers based on their reliability and expertise level. Experiment results indicate that the rewards are paid in alignment with individual reliability and expertise level of the workers.

\section{REFERENCES}

[1] D. C. Shoup, "Cruising for parking," Transport Policy, vol. 13(6), pp. 479-486, 2006.

[2] A. O. Kotb, Y.-C. Shen et al., "iParker - A New Smart Car-Parking System Based on Dynamic Resource Allocation and Pricing," IEEE Trans. Intell. Transp. Syst., vol. 17(9), pp. 2637-2647, 2016.

[3] Parker, http://www.theparkerapp.com.

[4] ParkMe, http://www.parkme.com.

[5] SmartParking, https://www.smartparking.com/.

[6] T. Rajabioun and P. Ioannou, "On-Street and Off-Street Parking Availability Prediction Using Multivariate Spatiotemporal Models," IEEE Trans. Intell. Transp. Syst., 2015.

[7] S. Mathur, T. Jin, N. Kasturirangan, J. Chandrasekaran, W. Xue, M. Gruteser, and W. Trappe, "ParkNet: drive-by sensing of road-side parking statistics," in Proc. ACM MobiSys, 2010, pp. 123-136.

[8] R. Lu, X. Lin, H. Zhu, and X. Shen, "SPARK: A New VANET-Based Smart Parking Scheme for Large Parking Lots," in Proc. IEEE Conf. Comput. Commun. (INFOCOM), 2009, pp. 1413-1421.

[9] R. K. Ganti, F. Ye, and H. Lei, "Mobile crowdsensing: current state and future challenges," IEEE Comm. Mag., vol. 49(11), pp. 32-39, 2011.

[10] M. Xiao, J. Wu, L. Huang, Y. Wang, and C. Liu, "Multi-Task Assignment for CrowdSensing in Mobile Social Networks," in Proc. IEEE Conf. Comput. Commun. (INFOCOM), 2015, pp. 2227-2235.

[11] D. Wu, Y. Zhang, L. Bao, and A. C. Regan, "Location-Based Crowdsourcing for Vehicular Communication in Hybrid Networks," IEEE Trans. Intell. Transp. Syst., vol. 14(2), pp. 837-846, 2013.

[12] S. Nawaz et al., "ParkSense: a smartphone based sensing system for on-street parking," in Proc. ACM MobiCom, 2013, pp. 75-86.

[13] V. Coric and M. Gruteser, "Crowdsensing Maps of On-street Parking Spaces," in Proc. IEEE DCOSS, 2013, pp. 115-122.

[14] N. True, "Vacant parking space detection in static images," University of California, San Diego, vol. 17, 2007.

[15] Q. Wu, C. Huang, S.-y. Wang et al., "Robust parking space detection considering inter-space correlation," in Proc. IEEE Multimedia and Expo, 2007, pp. 659-662.

[16] R. Yusnita, F. Norbaya, and N. Basharuddin, "Intelligent parking space detection system based onimage processing," International Journal of Innovation, Management and Technology, vol. 3, no. 3, p. 232, 2012.
[17] J. Jermsurawong, M. U. Ahsan, A. Haidar, H. Dong, and N. Mavridis, "Car parking vacancy detection and its application in 24-hour statistical analysis," in Proc. IEEE Frontiers of Information Technology (FIT), 2012, pp. 84-90.

[18] D. Zhang, H. Xiong, L. Wang, and G. Chen, "CrowdRecruiter: selecting participants for piggyback crowdsensing under probabilistic coverage constraint," in ACM UbiComp, 2014, pp. 703-714.

[19] P. Zhou, Y. Zheng, and M. Li, "How Long to Wait?: Predicting Bus Arrival Time with Mobile Phone based Participatory Sensing," in Proc. ACM MobiSys, 2012, pp. 379-392.

[20] Y. Gao, W. Dong, K. Guo et al., "Mosaic: A low-cost mobile sensing system for urban air quality monitoring," in Proc. IEEE Conf. Comput. Commun. (INFOCOM), 2016, pp. 1-9.

[21] J. Wan, J. Liu, Z. Shao et al., "Mobile crowd sensing for traffic prediction in internet of vehicles," Sensors, vol. 16(1), 2016.

[22] V. Raykar, S. Yu, L. Zhao, G. Valadez, L. B. C. Florin, and L. Moy, "Learning from crowds," The Journal of Machine Learning Research, vol. 11, pp. 1297-1322, 2010.

[23] S. Butakov, "Crowdcrawling approach for community based plagiarism detection service," in Proc. ACM WWW, 2014, pp. 1093-1096.

[24] M. H. Cheung, "Distributed time-sensitive task selection in mobile crowdsensing," in Proc. ACM MobiHoc, 2015, pp. 157-166.

[25] S. He, D. H. Shin, J. Zhang et al., "Distributed time-sensitive task selection in mobile crowdsensing," in Proc. IEEE Conf. Comput. Commun. (INFOCOM), 2014, pp. 745-753.

[26] D. Peng, F. Wu, and G. Chen, "Pay as How Well You Do: A Quality Based Incentive Mechanism for Crowdsensing," in ACM MobiHoc, 2015, pp. 177-186.

[27] D. Wang, L. Kaplan, H. Le et al., "On truth discovery in social sensing: A maximum likelihood estimation approach," in Proc. IEEE Conf. Information Processing in Sensor Networks (IPSN), 2012, pp. 233-244.

[28] L. Su et al., "Generalized decision aggregation in distributed sensing systems," in Proc. IEEE Real-Time Sys. Symp., 2014, pp. 1-10.

[29] S. Yang, F. Wu, S. Tang et al., "On designing data quality-aware truth estimation and surplus sharing method for mobile crowdsensing," IEEE J. Sel. Areas Commun., vol. 35(4), pp. 832-847, 2017.

[30] J.-S. Lee and B. Hoh, "Sell your experiences: a market mechanism based incentive for participatory sensing," in Proc. IEEE PerCom, 2010, pp. $60-68$.

[31] L. Gao, F. Hou, and J. Huang, "Providing long-term participation incentive in participatory sensing," in Proc. IEEE Conf. Comput. Commun. (INFOCOM), 2015, pp. 2803-2811.

[32] T. Luo, H.-P. Tan, and L. Xia, "Providing long-term participation incentive in participatory sensing," in Proc. IEEE Conf. Comput. Commun. (INFOCOM), 2014, pp. 127-135.

[33] T. Luo, S. S. Kanhere, J. Huang et al., "Sustainable incentives for mobile crowdsensing: Auctions, lotteries, and trust and reputation systems," IEEE Commun. Mag., vol. 55(3), pp. 68-74, 2017.

[34] T. Luo, S. S. Kanhere, H. P. Tan et al., "Crowdsourcing with tullock contests: A new perspective," in Proc. IEEE Conf. Comput. Commun. (INFOCOM), 2015, pp. 2515-2523.

[35] C.-J. Ho, A. Slivkins, S. Suri, and J. W. Vaughan, "Incentivizing high quality crowdwork," in Proc. ACM WWW, 2015, pp. 419-429. 
[36] D. Wang, T. F. Abdelzaher, L. M. Kaplan, and C. C. Aggarwal, "Recursive Fact-Finding: A Streaming Approach to Truth Estimation in Crowdsourcing Applications," in IEEE ICDCS, 2013, pp. 530-539.

[37] A. W. Van der Vaart, "Asymptotic statistics'," Cambridge series in statistical and probabilistic mathematics, vol. 3, 2000.

[38] Q. Liu, A. T. Ihler, and M. Steyvers, "Scoring workers in crowdsourcing: How many control questions are enough?" in Advances in Neural Information Processing Systems (NIPS), 2013, pp. 1914-1922.

[39] R. T. Fielding, "Rest: architectural styles and the design of networkbased software architectures," University of California, 2000.

[40] Foursquare Venue API, https://developer.foursquare.com/docs/api/.

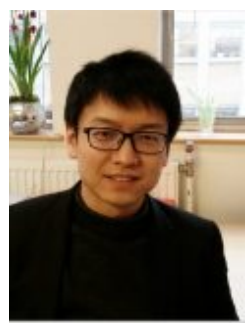

Fengrui Shi received the MSc in Electrical Engineering and Information Technology from ETH Zurich where he studied computer engineering with a focus on mobile systems. He received the BSc in Electronics and Information Engineering from Zhejiang University. He is a Ph.D. student in the Department of Computing at Imperial College London. He focuses on fundamentals issues of mobile crowdsensing systems such as data collection, analytics, resource allocation and incentivisation.

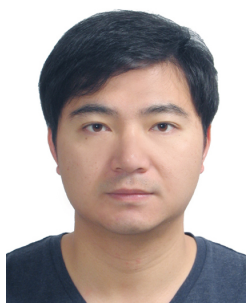

Di Wu received the M.S. and Ph.D. degree in Computer Science from the University of California, Irvine, USA in 2012 and 2013 respectively. He is currently an associate professor in the Department of Computer Engineering at Hunan University, China $\mathrm{He}$ is also an adjunct researcher at the University of California Transportation Center. His research interests include wireless networks and mobile computing, Internet-of-things, cyber-physical systems, smart cities and big data. He was a researcher at the Intel Collaborative Research Institute for Sustainable Connected Cities at Imperial College London, a staff research associate at the University of California, Irvine, a visiting researcher at IBM Research, and a research associate at the Stanford Research Institute. He has actively served on many conference committees and is an editor of the IEEE Transactions on Intelligent Transportation Systems. He is a member of IEEE and ACM.

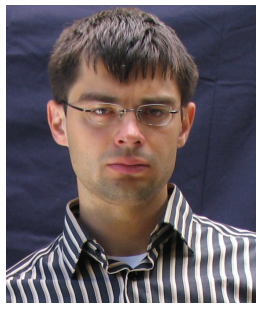

Dmitri I. Arkhipov received the B.S. degree in Information and Computer Science, the M.S. degree in Computer Science, and the Ph.D. degree in Computer Science from the University of California, Irvine in 2009, 2012 and 2016 respectively. He is currently a postdoctoral researcher in the Department of Computer Science at the University of California, Irvine. His research interests include parallel and distributed systems, large scale combinatorial optimization, and social networks.

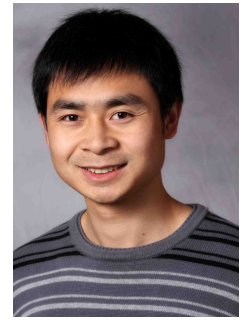

Qiang Liu received the B.S. degree in Information and Computation Science from the Beihang University, China in 2008. He received the M.S. degree in Mathematical, Computational and Systems Biology, and the Ph.D. degree in Computer Science from the University of California, Irvine, USA in 2009 and 2014 respectively. He is currently an assistant professor in the Department of Computer Science at Dartmouth College. His research area is machine learning and statistics, with interests spreading over the pipeline of data collection (mainly crowdsourcing), learning, inference, decision making, and various applications under the framework of probabilistic graphical models. He was a research intern at Microsoft Research Redmond and postdoctoral researcher at MIT CSAIL.

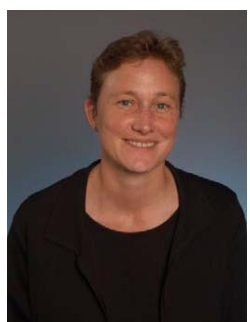

Amelia C. Regan received a B.A.S. degree in Systems Engineering from the University of Pennsylvania, an M.S. in Applied Mathematics from the Johns Hopkins University and an M.S. and Ph.D. in Transportation Systems Engineering from the University of Texas, Austin. She is a professor of computer science and transportation systems engineering at the University of California, Irvine. Her research is focused on algorithm development for optimization of transportation and communication systems. She has also taught short courses at the Athens University of Business and Economics and the National Technical University of Denmark and was an operations research analyst with the Association of American Railroads and United Parcel Service earlier in her career

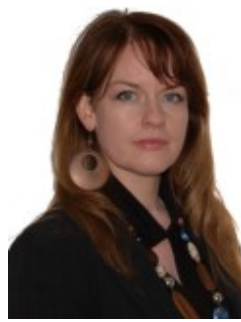

Julie A. McCann is a professor in computer systems with Imperial College. Her research centres on highly decentralized and self-organizing scalable algorithms for spatial computing systems, e.g., wireless sensing networks. She leads both the Adaptive Embedded Systems Engineering Research Group and the Intel Collaborative Research Institute for Sustainable Cities, and is currently working with NEC and others on substantive smart city projects. She has received significant funding though bodies such as the United Kingdoms EPSRC, TSB, and NERC as well as various international funds, and is an elected peer for the EPSRC. She has actively served on, and chaired, many conference committees and is currently associative editor of the ACM Transactions on Autonomous and Adaptive Systems. She is a fellow of the British Computer Society (BCS). 